\title{
Effect of concurrent resistance and sprint training on body composition and cardiometabolic health indicators in masters cyclists
}

\author{
Luke Delvecchio, ${ }^{1, *}$, Peter Reaburn², Gail Trapp ${ }^{3}$, Marko T. Korhonen ${ }^{3}$ \\ ${ }^{1}$ School of Medical and Applied Sciences, Central Queensland University, Rockhampton, Australia \\ ${ }^{2}$ School of Medical Sciences, University of New South Wales, Sydney, Australia \\ ${ }^{3}$ Gerontology Research Centre, Department of Health Sciences, University of Jyvaskyla, Finland
}

In older previously sedentary individuals endurance training imposes a more effective stimulus to enhance cardiometabolic health compared with resistance or sprint training. We examined the effect of replacing a portion of endurance training with combined resistance and/or sprint training and how this influences cardiometabolic health indicators in masters endurance cyclists. Twenty-seven well-trained male road cyclists ( $53.7 \pm 8.2$ years) were allocated to a resistance and track sprintcycling training group ( $R T C, n=10)$, an endurance and track sprint-cycling group ( $E T C, n=7$ ) or a control endurance group (CTRL, $n=10$ ). Both the RTC and ETC groups completed a 12-week intervention of specific training while the CTRL group maintained their endurance training load. Lower limb lean mass (LLM), trunk fat mass (TFM), fasting blood glucose (FBG), total cholesterol (TC), triglycerides (TG), systolic blood pressure (SBP), and diastolic blood pressure (DBP) were measured before and after the intervention period. TFM decreased for all groups $(P<0.05)$ while LLM significantly increased for RTC and ETC groups $(P<0.05)$. No significant between group or time effects were observed for FBG, TC, TG, SBP, or DBP. The results suggest that replacing a portion of endurance training with 12 weeks of ETC or RTC training favourably affects body composition by lowering TFM and increasing LLM without negatively affecting cardiometabolic health indicators in welltrained masters endurance cyclists.

Keywords: Aging, Resistance training, Cycling, Blood pressure, Blood lipids

\section{INTRODUCTION}

Normal aging is typically associated with unfavourable changes in serum lipids and blood pressure levels, both of which increase the risk of cardiometabolic diseases such as atherosclerotic vascular disease and type 2 diabetes (Buitrago-Lopez et al., 2011). However, these negative changes in the metabolic profile are determined not only by biological aging itself but also lifestyle factors such as lack of exercise and a poor diet (Gaesser et al., 2011).

Previous studies suggests that resting blood pressure of middle-aged and older endurance runners are at optimal levels $(\leq 120 / 80 \mathrm{mmHg})$ and lower than in both age-matched strength and sprint athletes (Kusy and Zieliński, 2015) and inactive controls (Buyukyazi, 2005; Cornelissen et al., 2009; Hernelahti et al., 2002). Moreover, previous studies have shown lower blood glucose, total cholesterol (TC), and triglyceride levels in older endurance-trained athletes than age-matched non-athletic controls (Mikkelsen et al., 2013). Taken together, these data suggest endurance training may explain the lower blood pressure and lower blood lipid levels observed in older endurance athletes.

Despite the widely known performance and cardiometabolic benefits of endurance training, previous research has reported masters athletes involved in non-weight-bearing endurance sports such as cycling and swimming have lower bone mineral density
${ }^{*}$ Corresponding author: Luke Del Vecchio (iD http://orcid.org/0000-0001-7889-0232 School of Medical and Applied Sciences, Central Queensland University, Rockhampton, Australia

Tel: +61-7-4923-2427, Fax: +61-7-4930-9565, E-mail: 1.delvecchio@cqu.edu.au Received: July 2, 2016 / Accepted: September 19, 2016
This is an Open Access article distributed under the terms of the Creative Commons Attribution Non-Commercial License (http://creativecommons.org/licenses/by-nc/4.0/) which permits unrestricted non-commercial use, distribution, and reproduction in any medium, provided the original work is properly cited. 
compared with other athletes and even nonactive controls (Nichols et al., 2003; Rector et al., 2008). As a result of these findings, masters endurance cyclists may need to incorporate resistance training into their endurance training regimes to maintain optimal bone density as they age (Bolam et al., 2015; Hinton et al., 2015). In addition to resistance training, many road cyclists also incorporate high intensity sprint training to further improve road cycle-racing race performance where 'attacks' during a road race and sprint finishes are major factors in successful road cycle-racing racing. However, it is possible that replacing a portion of endurance training with strength or sprint training may lead to a reduction in endurance training volume which may negatively affect the cardiometabolic profile of masters road cyclists. Indeed, previous research has shown that reductions in endurance training volume lead to increased cardiometabolic risk in both younger runners (Sutherland et al., 1981) and masters cyclists (Giada et al., 1995). In contrast, recent evidence suggests that positive metabolic and musculoskeletal adaptations in healthy older adults occur from short-term resistance and sprint training if the intensity of that training is high (Bell et al., 2015; Nederveen et al., 2015). Therefore, the purpose of this study was to determine if replacing a portion of endurance training with combined resistance and sprint training or sprint training alone will negatively affect cardiometabolic health indicators in masters endurance cyclists.

\section{MATERIALS AND METHODS}

\section{Participants}

The study was approved by the Central Queensland University Human Research Ethics Committee. Twenty seven male masters endurance cyclists ( $53.7 \pm 8.2$ years) with no background of resistance training were recruited and provided written informed consent. The subjects were required to be involved in regular cycling training and competition for a minimum of 2 years and to be achieving a minimum of $8 \mathrm{hr}$ of endurance cycling training per week. All subjects underwent pre-exercise screening to ensure they had no established cardiovascular, metabolic or respiratory disease (nor) signs (or) symptoms of these conditions (Norton, 2005).

Random allocation of participants into training groups was not possible as a result of the majority of participants having both work and family commitments that limited their availability to participate in the RTC or ETC training programs. As a result, subjects were allocated to either a control group (CTRL, $\mathrm{n}=10$ ), an endurance and track sprint-cycling group (ETC, $n=7$ ) or resistance and track sprint-cycling training group $(\mathrm{RTC}, \mathrm{n}=10)$ based
Table 1. Physical characteristics and training hours per week of the groups

\begin{tabular}{lccc}
\hline Variable & RTC group $(n=10)$ & ETC group $(n=7)$ & CTRL group $(n=10)$ \\
\hline Age $(\mathrm{yr})$ & $53.5 \pm 9.3$ & $49.4 \pm 4.8$ & $56.9 \pm 8.6$ \\
Stature $(\mathrm{m})$ & $1.80 \pm 0.1$ & $1.80 \pm 0.1$ & $1.75 \pm 0.1$ \\
Body mass $(\mathrm{kg})$ & $81.9 \pm 6.1$ & $78.5 \pm 6.1$ & $83.5 \pm 10.0$ \\
Training hours (hr/wk) & $8.2 \pm 1.0$ & $8.1 \pm 1.3$ & $8.0 \pm 1.2$ \\
\hline
\end{tabular}

Values are presented as mean \pm standard deviation.

RTC, resistance and track sprint-cycling group; ETC, endurance and track sprint-cycling group; CTRL, control endurance group.

on their availability. Participants were encouraged and agreed to avoid changes in their diet or lifestyle over the intervention period. The physical characteristics of each group are shown in Table 1.

\section{Protocol}

Subjects attended the laboratory following an overnight fast and did not consume caffeine the morning of the testing sessions carried out between 07:00 a.m. and 09:00 a.m. hr. Pre- and postintervention testing included, in order, anthropometric measures, Dual energy X-ray absorptiometry (DEXA), fasting blood glucose (FBG), fasting blood lipids, resting blood pressure and determination of peak aerobic power $\left(\mathrm{VO}_{\text {2peak }}\right)$ on a cycle ergometer. All measurements were performed by the same trained observer.

\section{Anthropometry measures}

Stature $(\mathrm{m})$ and body mass $(\mathrm{kg})$ were measured with a stadiometer and medical scales (Seca, Birmingham, UK) with participant's unshod and wearing cycling apparel.

\section{DEXA scanning}

DEXA (Hologic Discovery-W, Bedford, MA, USA) was used to measure trunk fat mass (TFM) and lower limb lean mass (LLM). A single trained DEXA technician performed all DEXA measurements. The trunk region consists of the area bordered by a horizontal line below the chin, vertical borders lateral to the ribs and oblique lines passing through the femoral necks. The leg region includes all tissue below these oblique lines.

\section{Blood measures}

Subjects were required to fast for $8 \mathrm{hr}$ prior to the blood tests. FBG, TC, and triglycerides (TG) were measured via a $30-\mu \mathrm{L}$ capillarized blood sample taken from each subject's fingertips. For the analysis of FBG, TC, and TG a sample of whole blood was collected into a capillary pipette and applied to the Reflotron reagent strip (Roche Diagnostics, Sydney, Australia). Blood samples were processed using a Reflotron Plus reflectance photometer (Hoffman 
La Roche Ltd., Basel, Switzerland). Coefficient of variation for FBG is $2.5 \%$, TC is $1.2 \%$, and TG is $2.9 \%$ (Roche Diagnostics, Sydney, Australia).

\section{Resting blood pressure}

Resting blood pressure was measured upon arrival at the laboratory and after the subjects had undertaken 10 min of seated rest in quiet and comfortable conditions. Blood pressure was measured using a standard mercury hand held sphygmomanometer (Nova-Presameter, Riester, Jungingen, Germany) using the standard guidelines established by the American Heart Association (Perloff et al., 1993). Blood pressure was obtained in triplicate for both arms and each measurement was separated from the next by a 1to 2-min resting period. The blood pressure obtained from the arm with the highest reading was used for statistical analysis.

$\mathrm{VO}_{\text {2peak }}$

A graded maximal exercise test to measure $\mathrm{VO}_{\text {2peak }}$ was completed on an electrically-braked, computer controlled cycle ergometer (Velotron Dynafit Pro, RaceMate, Seattle, WA, USA). Gas analysis was undertaken using a Fitmate Pro (Cosmed, Rome, Italy) (Brisswalter and Tartaruga, 2014) following a 5-min warmup at 30-W cycling and a pedalling cadence of $90 \mathrm{rpm}$ throughout the test. The work increments for each one-minute stage were $15 \mathrm{~W}$. The test ceased when no significant increase in $\mathrm{O}_{2}$ uptake with an increase in work rate and/or volitional exhaustion (Schell and Leelarthaepin, 1990). The test was followed by a 5 -min cool down at a self-selected intensity and cadence.

\section{Endurance and track sprint-cycling program}

Both RTC and ETC groups replaced two of their usual weekly endurance cycling training sessions with two group track-cycling session per week lasting approximately $90 \mathrm{~min}$, separated by 48 $\mathrm{hr}$. The sprint cycling program was performed in the evening at an outdoor cycling velodrome. The training program was designed in consultation with an accredited track cycling coach and supervised by the same coach for each session. The sprint cycling training sessions consisted of a five to 10-min warm-up of 10-15 laps at a self-selected pace after which subjects performed 1-3 sets $\times 1-3$ repetitions of maximal effort sprints ranging in distance from 65 to $330 \mathrm{~m}$ (sprint times ranged from 6 to $30 \mathrm{sec}$ ) with 2-3 min of active recovery between repetitions and 10- to 15-min passive rest between sets. At the completion of the track training session subjects performed a 5- to 10-min cool down up of 10-15 laps of the velodrome at a self-selected pace. Selected strategies to achieve increased maximum speed and acceleration capabilities included 'Over Gear Training' with a gear that is larger than the athlete's typical race gear. Furthermore, the sprint cycling program also incorporated an ascending progressive gear overload method where the gearing size increased progressively following each individual sprint. The overall training adherence rate calculated as a percentage of the total training sessions successfully completed was $87 \% \pm 4 \%$ for all sprint cycling training across the 12-week study period with no differences between the RTC and ETC groups.

\section{Resistance training and track sprint-cycling program}

The RTC group replaced four of their usual weekly endurance cycling training sessions with two evening group track sprint-cycling training sessions as described above, and two morning gymbased group resistance training sessions per week. All four training sessions were supervised by an accredited strength and conditioning coach. Resistance training sessions were conducted on alternate days to the track sprint training days. During each resistance training session participants completed exercises in the following order: double- and single-leg hopping (2-3 sets of 10-20 hops), box jumps, leg press throws, single-leg leg presses, seated hip flexions, leg curls, leg extensions, seated calf-raises, supine hip extensions, chest presses, bench rows, abdominal curl ups and lower back extensions. Recovery time of 2 min between sets and exercises was strictly controlled with the resistance training sessions lasting approximately $90 \mathrm{~min}$. The progressive resistance training program was periodised to reduce both the potential for overtraining and to optimise neuromuscular adaptation. Subjects completed electronic training logs describing all their training parameters (number of repetitions, sets, loads, track and road training distances, track sprint cycling times) to monitor progress and to provide motivation for maximal effort during the training program. The overall training adherence rate, calculated as a percentage of training sessions successfully completed was $85 \% \pm 4 \%$ for track sprint-cycling training and $82 \% \pm 5 \%$ for resistance training across the 12-week study period.

\section{Control group}

The CTRL group were asked to maintain their normal endurance cycling training for the 12 -week intervention period.

\section{Statistical analysis}

A three (ETC, RTC, CTRL) $\times$ two (pre, post) repeated measures analysis of variance was used to contrast dependent variables of in- 
Table 2. DEXA, fasting blood glucose, blood lipids and $\mathrm{VO}_{2 \text { peak }}$ changes in $\mathrm{RTC}, \mathrm{ETC}$, and CTRL groups following the 12-week intervention

\begin{tabular}{|c|c|c|c|c|c|c|c|c|c|}
\hline \multirow{2}{*}{ Variable } & \multicolumn{3}{|c|}{ RTC group $(n=10)$} & \multicolumn{3}{|c|}{ ETC group $(n=7)$} & \multicolumn{3}{|c|}{ CTRL group $(n=10)$} \\
\hline & Pre & Post & Change (\%) & Pre & Post & Change (\%) & Pre & Post & Change (\%) \\
\hline TFM (kg) & $7.7 \pm 2.7$ & $7.3 \pm 2.1$ & -5.1 & $6.7 \pm 1.1$ & $6.0 \pm 0.9$ & -10.4 & $8.7 \pm 1.1$ & $8.5 \pm 1.3$ & -2.2 \\
\hline LLM (kg) & $17.4 \pm 1.8$ & $18.0 \pm 2.3^{a)}$ & 3.4 & $17.0 \pm 1.5$ & $17.6 \pm 1.4^{\mathrm{al}}$ & 3.5 & $16.0 \pm 2.0$ & $16.0 \pm 1.9$ & 0.0 \\
\hline $\mathrm{FBG}(\mathrm{mmol} / \mathrm{L})$ & $5.1 \pm 0.3$ & $5.0 \pm 1.2$ & -2.7 & $5.3 \pm 0.7$ & $4.64 \pm 0.9$ & -12.6 & $5.1 \pm 0.6$ & $5.2 \pm 0.8$ & 0.7 \\
\hline $\mathrm{TC}$ (mmol/L) & $4.2 \pm 1.0$ & $3.9 \pm 1.1$ & -7.1 & $4.7 \pm 0.7$ & $4.2 \pm 1.2$ & -10.6 & $4.8 \pm 1.1$ & $4.5 \pm 1.6$ & -6.25 \\
\hline $\mathrm{TG}$ (mmol/L) & $0.92 \pm 0.2$ & $0.90 \pm 0.25$ & -2.2 & $1.2 \pm 0.4$ & $1.0 \pm 0.5$ & -16.7 & $1.9 \pm 1.8$ & $1.6 \pm 0.60$ & -15.8 \\
\hline $\mathrm{SBP}(\mathrm{mmHg})$ & $122 \pm 5.9$ & $119 \pm 6.5$ & -2.4 & $129 \pm 9.0$ & $121 \pm 9.3$ & -6.2 & $136 \pm 12.2$ & $138 \pm 21.8$ & 1.5 \\
\hline $\mathrm{DBP}(\mathrm{mmHg})$ & $79 \pm 5.5$ & $78 \pm 6.5$ & -1.2 & $80 \pm 4.1$ & $78 \pm 6.9$ & -2.5 & $87 \pm 7.2$ & $88 \pm 5.8$ & 1.1 \\
\hline $\mathrm{VO}_{2 \text { peak }}(\mathrm{mL} / \mathrm{kg} / \mathrm{min})$ & $46.7 \pm 9.3$ & $45.6 \pm 8.4$ & -2.3 & $54.0 \pm 10.2^{\text {b) }}$ & $51.9 \pm 7.1$ & -3.9 & $36.9 \pm 9.2^{b l}$ & $38.4 \pm 6.9$ & 1.0 \\
\hline
\end{tabular}

Values are presented as mean \pm standard deviation.

DEXA, dual energy X-ray absorptiometry; $V_{2 \text { peak, }}$ peak aerobic power; RTC, resistance and track sprint-cycling group; ETC, endurance and track sprint-cycling group; CTRL, control endurance group; Change, percentage change from pre to post training; TFM, trunk fat mass; LLM, lower limb lean mass; FBG, fasting blood glucose; TC, total cholesterol; TG, triglycerides; SBP, systolic blood pressure; DBP, diastolic blood pressure.

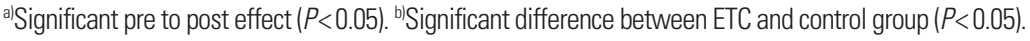

terest. If a main effect was observed, a Tukey post hoc test was undertaken to identify the source of the differences. A $P$-value of $<0.05$ was considered to be statistically significant. Cohen conventions for effect size (ES) were used for interpretation where ES= $0.2,0.5$, and 0.8 are considered as small, medium and large, respectively (Cohen, 2013). IBM SPSS Statistics ver. 20.0 (IBM Co., Armonk, NY, USA) software was used for all statistical analyses.

\section{RESULTS}

Table 2 shows the changes in each of the variables of interest for each of the groups over the 12-week intervention period.

\section{Trunk fat mass}

A significant effect of time was observed for TFM $(P<0.01)$. However, no significant between group effects were observed for TFM $(P=0.49)$ following the exercise training period. ES analysis revealed that 12 weeks of RTC training had no effect on TFM in the RTC group ( $E S=-0.16$ ) or CTRL group $(E S=-0.16)$. However, 12 weeks of ETC training had a moderate, negative effect on TFM in the ETC group $(E S=-0.69)$. There was no effect on TFM following the exercise training period observed in the control group $(\mathrm{ES}=-0.16)$.

\section{Lower LLM}

LLM increased significantly in both the RTC group $(P=0.01)$ and the ETC group $(P=0.01)$ with no significant between-group differences observed for $\operatorname{LLM}(P=0.89)$. Twelve weeks of RTC training had a small effect on LLM in the RTC group ( $E S=0.29$ ).
In contrast, 12 weeks of ETC training had a moderate effect on LLM in the ETC group (ES=0.41). There was no effect on LLM following the exercise training period observed in the control CTRL group $(E S=0.00)$.

\section{Fasting blood glucose}

There were no significant effects of time $(P=0.35)$ or between group differences observed for FBG $(P=0.46)$ following the study intervention period. ES analysis revealed RTC training had no effect on FBG in the RTC group (ES=-0.16). In contrast, 12 weeks of ETC training had a large effect $(E S=-0.83)$ on FBG in the ETC group. There was no effect on FBG following the exercise training period observed in the control CTRL group $(E S=-0.05)$.

\section{Total cholesterol}

There were no significant effects of time $(P=0.06)$ or between group differences $(P=0.82)$ observed for TC following the intervention. ES analysis revealed 12 weeks of RTC training had a small effect on ETC in the RTC group $(E S=-0.30)$. In contrast, 12 weeks of ETC training had a moderate effect on ETC in the ETC group ( $E S=-0.40)$. There was a small effect on TC following the exercise training period observed in the control group CTRL $(\mathrm{ES}=-0.21)$.

\section{Triglycerides}

There were no significant effects of time $(P=0.51)$ or between group differences $(P=0.22)$ observed for TG following the study period. ES analysis revealed 12 weeks of RTC training had no effect on TG in the RTC group (ES=-0.08). In contrast, 12 weeks 
of ETC had a small effect on TG following 12 weeks of ETC training in the ETC group $(E S=-0.30)$. There was a small effect on TG following the exercise training period observed in the CTRL group $(E S=-0.22)$.

\section{Blood pressure}

There were no significant effects of time $(P=0.34)$ or between group differences $(P=0.28)$ observed for SBP following the study period. ES analysis revealed 12 weeks of RTC training had a moderate effect on SBP following 12 weeks of RTC training (ES= -0.48). In contrast, a 12 weeks of ETC had a large effect on SBP following 12 weeks of ETC training in the ETC group (ES = -0.87). There was no effect on SBP following the exercise training period observed in the control CTRL group $(E S=0.11)$.

There were also no significant effects of time $(P=0.32)$ or between group differences $(P=0.43)$ observed for DBP following the study period. ES analysis revealed 12 weeks of RTC training had no effect on DBP in the RTC group (ES=-0.16). In contrast, 12 weeks of ETC training had a small effect on DBP in the ETC group $(\mathrm{ES}=-0.36)$. There was no effect on DBP following the exercise training period observed in the control CTRL group (ES= $0.15)$.

\section{$\mathrm{VO}_{\text {2peak }}$ (peak aerobic power)}

There was a significant between group difference in pre $\mathrm{VO}_{2 \text { peak }}$ $(P=0.01)$. Pre $\mathrm{VO}_{2 \text { peak }}$ was significantly greater in the ETC, compared to the CTRL group $(P=0.01)$ (Table 2$)$. There were no significant effects of time $(P=0.73)$ or between group differences $(P=0.60)$ observed for $\mathrm{VO}_{2 \text { peak }}$ following the study period. ES analysis revealed RTC training had no effect on $\mathrm{VO}_{2 \text { peak }}$ in the RTC group (ES=-0.12). In contrast, 12 weeks of ETC training had a small effect on $\mathrm{VO}_{2 \text { peak }}$ in the ETC group $(\mathrm{ES}=-0.23)$. There was no effect on $\mathrm{VO}_{2 \text { peak }}$ following the exercise training period observed in the control group ( $\mathrm{ES}=0.18$ ).

\section{DISCUSSION}

Recent evidence suggests that short-duration sprint and/or resistance training may lead to positive adaptations in cardiometabolic risk factors if the intensity of exercise is high (Bell et al., 2015; Kusy and Zieliński, 2015). This evidence supports the long held position that endurance training leads to significant improvements in the cardiometabolic risk profile in previously sedentary older individuals. In the present study we investigated whether the replacement a portion of endurance training by resis- tance and sprint exercise might influence body composition and cardiometabolic health indicators in endurance-trained masters cyclists. The major finding of the present study was that TFM was significantly reduced in both the ETC and RTC groups. Moreover, 12 weeks of RTC and ETC significantly increased LLM. However, there were no significant changes or between group differences in the important cardiometabolic health indicators of FBG, TC, TG, SBP, or DBP. Therefore, the current data supports the hypothesis that 12 weeks of RTC or ETC will not negatively affect cardiometabolic health indicators in masters endurance cyclists.

TFM is a well-recognised and independent risk factor for cardiometabolic disease (Hu et al., 2011). Thus, the significant reduction in TFM observed in the ETC and RTC groups of the present study suggest the addition of high intensity sprint and/or resistance training may lower the risk of cardiometabolic disease in endurance-trained masters cyclists. Indeed, in the present study, 12 weeks of ETC reduced TFM by $10.4 \%$ accompanied by a moderate ES (-0.69). Moreover, the RTC group reduced TFM by $5.1 \%$ while the CTRL group reduced TFM by $2.2 \%(-0.16)$. These findings are in agreement with earlier findings by Treuth et al. (1994) who reported a significant reduction in TFM following 16 weeks of resistance training performed three times per week in a group of healthy, older males aged 50-75 years. More recently, Sillanpää et al. (2009) also reported a significant reduction in TFM following 21 weeks of combined resistance and endurance training performed four times per week in a group of middle-aged females aged $48.9 \pm 6.8$ years. However, our study is the first to have investigated the effects of resistance and/or sprint training on TFM in healthy older adults who are also masters athletes. Taken together, the present and previous results suggest ETC training may be more beneficial for reducing TFM than RTC or endurance cycling training alone in masters endurance cyclists.

The present study also observed a significant increase in LLM following 12 weeks of RTC and ETC training. These findings are in agreement with previous studies that have reported significant increases in lean mass in response to a combined resistance and sprint training intervention in masters sprint runners (Cristea et al., 2008; Reaburn et al., 1994). For example, Cristea et al. (2008) reported a significant increase in LLM in a group of sprint-trained male masters sprint runners who completed a 20 -week progressive resistance training program. The loss of muscle mass with age is an independent risk factor for cardiometabolic disease (Dominguez and Barbagallo, 2007). Masters athletes, like their sedentary agematched counterparts are susceptible to an age-related decline in 
muscle mass (Reaburn and Dascombe, 2009) which may thus increase their risk of cardiometabolic disease. In the present study, RTC training had a small effect on LLM in the RTC group with a moderate effect on LLM observed in the ETC group. Recent research suggests sprint training may positively affect lean mass in healthy but previously untrained older adults (Bell et al., 2015; Nederveen et al., 2015). Taken together with these previous findings, the present data suggest sprint training may induce muscle hypertrophy in masters athletes which subsequently may reduce the risk for cardiometabolic disease. Moreover, the present findings suggest the increased muscle hypertrophy from augmenting endurance training with sprint or resistance training does not appear to negatively impact on important cardiometabolic risk factors.

In the present study we also examined the effects of 12 weeks of ETC and RTC training on a number of commonly measured cardiometabolic risk factors including FBG, total cholesterol, TG, and blood pressure. In summary, the current data suggests RTC and ETC had no negative effect on any of the cardiometabolic risk factors when an older endurance athlete has a significant volume of their endurance training replaced by either sprint training alone or concurrent sprint and resistance training.

Hyperglycaemia is a widely acknowledged risk factor for cardiometabolic disease (Brunzell et al., 2008). The present study observed that 12 weeks of RTC or ETC did not negatively affect FBG levels in masters endurance cyclists who replaced a significant portion of their normal endurance training with sprint and/ or resistance training. These data are in agreement with similar studies that have observed no effect of resistance training on FBG in healthy older adults (Ferrara et al., 2004; Zachwieja et al., 1996). In the present study, 12 weeks of ETC nonsignificantly reduced FBG by $12.6 \%$ but was accompanied by a large ES (-0.83) in the ETC group. Previous research examining the effect of sprint and endurance training in masters athletes suggest that long-term sprint training may not have a favourable effect on glucose metabolism (Kusy and Zieliński, 2015; Kusy et al., 2013). Importantly, the FBG of both the RTC and ETC groups were within the acceptable range for FBG (World Health Organization, 2006) suggesting that RTC and ETC training maintains FBG within healthy ranges. Taken together, the results of the current study suggest that 12 weeks of RTC or ETC does not negatively affect FBG in masters endurance cyclists who replace part of their normal endurance training with sprint-only or concurrent sprint and resistance training.

Elevated TC increases the risk for cardiometabolic disease, particularly with age (Campesi et al., 2016). The present study is the first to examine the effect of ETC and RTC on TC in masters athletes. We observed that 12 weeks of ETC or RTC did not negatively affect TC levels in masters endurance cyclists who lowered their endurance training volume to accommodate the ETC or RTC training. These findings are consistent with previous studies examining the effect of sprint cycling training on blood lipids in overweight and previously sedentary middle-aged men (Moreira et al., 2008; Wallman et al., 2009). Previous research has also shown that masters athletes ( $45.9 \pm 4.8$ years) who regularly participate in field sports such as soccer or hockey had significantly lower TC levels than age-matched sedentary controls (Dey et al., 2002). These data suggest that sports which involve repeated powerful muscular actions and sprinting may exert a positive effect on TC in masters athletes. The findings of the present study support this suggestion with a small effect $(-0.10)$ and $10.6 \%$ reduction in TC following 12 weeks of track sprint cycling training. Taken together, this data suggests reducing replacing a portion of endurance training with track-sprint training does not negatively affect T-C in masters endurance cyclists.

In the present study, TC was reduced by $7.1 \%$ within the RTC group, but these changes were not significant. However, the reductions in TC observed in the present study may be clinically relevant since as little as a one percent reduction in TC has been shown to be associated with a two to three percentage reduction in the incidence of coronary heart disease (Law et al., 1994). Therefore, our data suggests both ETC and RTC training may be used by older populations to lower TC and impact on cardiometabolic health. To date, no research has investigated the effects of resistance training on $\mathrm{TC}$ in masters athletes. The present findings are in agreement with previous research which has shown in untrained older men, high intensity resistance training favourably effects TC levels (Hagerman et al., 2000; Joseph et al., 1999; Leenders et al., 2013). In addition, previous research (Martins et al., 2010) has reported moderate to high intensity resistance training also induces favourable changes in TC in healthy older males ( $76 \pm 8.0$ years). Taken together, these results again suggest TC is not negatively influenced by RTC training in masters endurance cyclists.

Elevated TG are an independant risk factor for cardiometabolic disease (Pirillo et al., 2014). In the current study, 12 weeks of either ETC training or RTC training had no significant effect on TG in masters endurance cyclists. These results are in agreement with previous studies that have reported no significant reduction in TG in response to high intensity sprint training in overweight, middle-aged males and females (Moreira et al., 2008) and obese middle-aged males and females (Wallman et al., 2009). Moreover, 
previous studies have also reported no significant reduction of resistance training alone on TG in healthy older men (Hagerman et al., 2000) and overweight older males and females (Joseph et al., 1999).

In summary, the lack of a significant affect of ETC and RTC on TC and TG in the current study may be attributed to the following factors. First, participants in both the training groups were already well-trained endurance cyclists and thus may have already lowered their lipids, a finding commonly observed following years of endurance training (Thompson et al., 1988; Wallman et al., 2009). Secondly, the small detraining effect on $\mathrm{VO}_{2 \text { peak }}$ observed in the RTC and ETC groups may be partly responsible for the small changes in TC and TG. For example, Giada et al. (1995) reported an 8-week detraining period significantly increased TG and decreased high-density lipoprotein cholesterol in conjuction with a significant decrease in $\mathrm{VO}_{2 \text { peak }}$ in a group of masters cyclists aged 50-65 years.

Hypertension is associated with several cardiometabolic disorders including diabetes and dyslipidaemia (Neves et al., 2013). In the current study, 12 weeks of RTC training reduced SBP by 3.0 $\mathrm{mmHg}$ and DBP by $1.0 \mathrm{mmHg}$ with these results accompanied by a moderate effect on SBP (-0.48) but no effect on DBP. To the best of our knowledge, no previous studies have investigated the effects of resistance training on blood pressure in masters athletes. However, previous research suggests moderate intensity resistance training has favourable effects on blood pressure in healthy older adults (Collier et al., 2008; Westcott et al., 2009). While the present changes were not statistically significant, a $5-\mathrm{mmHg}$ drop in SBP at a population level is associated with a $9 \%$ reduction in death due to coronary Heart Disease (Sharman and Stowasser, 2009). Thus, the current study demonstrates that RTC training may have the potential to further reduce BP and thus cardiometabolic risk in masters endurance cyclists.

The current study observed no negative effect of ETC training on SBP or DBP in masters endurance cyclists. In the present study, 12 weeks of ETC training reduced SBP by $8 \mathrm{mmHg}$ and DBP by $2.2 \mathrm{mmHg}$ nonsignificantly with but with these reductions complemented by a large effect on SBP $(-0.87)$ and a moderate effect on DBP (-0.65). However, the observed nonsignificant reductions in SBP observed in the present study may be clinically relevant since a reduction in SBP of $3 \mathrm{mmHg}$ for normotensive individuals such as those in the present study has been shown to reduce all-cause mortality by $4 \%$ at a population level (Collier et al., 2008).

Previous observational studies have suggested that sprint train- ing may reduce BP measures in aging athletes when comparing blood pressure values in masters track and field athletes with those from healthy, age-matched controls (Hernelahti et al., 2002; Kettunen et al., 2006).Therefore, the present and previous results suggest ETC training may be used in a prehypertensive older population to lower BP. In summary, the results of the current study suggest replacing a portion of endurance training with either ETC alone or RTC training does not have a negative effect on both SBP and DBP in masters endurance cyclists.

Finally, in the present study, we observed a small but nonsignificant decrease in $\mathrm{VO}_{2 \text { peak }}$ in the RTC group $(-2.3 \%)$ and the ETC group (-3.9\%). In contrast the CTRL group demonstrated a small nonsignificant increase $(1 \%)$. However, these changes are within standard error of measurement ranges for $\mathrm{VO}_{2 \text { peak }}$ testing both within our laboratory and Australian accredited exercise testing laboratories (Tanner and Gore, 2013). These results suggest that replacing a portion of endurance training with 12 weeks of either ETC or RTC training does not significantly reduce $\mathrm{VO}_{\text {2peak }}$, despite a reduction in overall endurance training volume.

The results of the present study suggest that 12 weeks of ETC or RTC training favourably affects body composition by lowering TFM and increasing LLM in well-trained masters endurance cyclists. These positive changes in body composition may lower the risk for cardiometabolic disease. Moreover, the present findings suggest that cardiometabolic health indicators including FBG, TC, TG, SBP, and DBP are unaffected by a reduction in endurance training volume in masters endurance cyclists who replace part of their endurance training volume and undertake resistance and/or sprint training.

\section{CONFLICT OF INTEREST}

No potential conflict of interest relevant to this article was reported.

\section{REFERENCES}

Bell KE, Séguin C, Parise G, Baker SK, Phillips SM. Day-to-day changes in muscle protein synthesis in recovery from resistance, aerobic, and high-intensity interval exercise in older men. J Gerontol A Biol Sci Med Sci 2015;70:1024-1029.

Bolam KA, Skinner TL, Jenkins DG, Galvão DA, Taaffe DR. The Osteogenic effect of impact-loading and resistance exercise on bone mineral density in middle-aged and older men: a pilot study. Gerontology 2015;62:22-32. 
Brisswalter J, Tartaruga MP. Comparison of COSMED'S FitMate ${ }^{\mathrm{TM}}$ and K4b2 metabolic systems reliability during graded cycling exercise. Scand J Clin Lab Invest 2014;74:722-724.

Brunzell JD, Davidson M, Furberg CD, Goldberg RB, Howard BV, Stein JH, Witztum JL. Lipoprotein management in patients with cardiometabolic risk: consensus conference report from the American Diabetes Association and the American College of Cardiology Foundation. J Am Coll Cardiol 2008;51:1512-1524

Buitrago-Lopez A, Sanderson J, Johnson L, Warnakula S, Wood A, Di Angelantonio E, Franco OH. Chocolate consumption and cardiometabolic disorders: systematic review and meta-analysis. BMJ 2011;343:d4488.

Buyukyazi G. Differences in blood lipids and apolipoproteins between master athletes, recreational athletes and sedentary men. J Sports Med Phys Fitness 2005;45:112-120.

Campesi I, Occhioni S, Tonolo G, Cherchi S, Basili S, Carru C, Zinellu A, Franconi F. Ageing/menopausal status in healthy women and ageing in healthy men differently affect cardiometabolic parameters. Int J Med Sci 2016;13:124-132.

Cohen J. Statistical power analysis for the behavioral sciences. Hoboken: Taylor and Francis; 2013.

Collier SR, Kanaley JA, Carhart R Jr, Frechette V, Tobin MM, Hall AK, Luckenbaugh AN, Fernhall B. Effect of 4 weeks of aerobic or resistance exercise training on arterial stiffness, blood flow and blood pressure in pre- and stage-1 hypertensives. J Hum Hypertens 2008;22:678686.

Cornelissen VA, Arnout J, Holvoet P, Fagard RH. Influence of exercise at lower and higher intensity on blood pressure and cardiovascular risk factors at older age. J Hypertens 2009;27:753-762.

Cristea A, Korhonen MT, Häkkinen K, Mero A, Alén M, Sipilä S, Viitasalo JT, Koljonen MJ, Suominen H, Larsson L. Effects of combined strength and sprint training on regulation of muscle contraction at the wholemuscle and single-fibre levels in elite master sprinters. Acta Physiol (Oxf) 2008;193:275-289.

Dey SK, Ghosh C, Debray P, Chatterjee M. Coronary artery disease risk factors \& their association with physical activity in older athletes. J Cardiovasc Risk 2002;9:383-392.

Dominguez LJ, Barbagallo M. The cardiometabolic syndrome and sarcopenic obesity in older persons. J Cardiometab Syndr 2007;2:183-189.

Ferrara CM, McCrone SH, Brendle D, Ryan AS, Goldberg AP. Metabolic effects of the addition of resistive to aerobic exercise in older men. Int J Sport Nutr Exerc Metab 2004;14:73-80.

Gaesser GA, Angadi SS, Sawyer BJ. Exercise and diet, independent of weight loss, improve cardiometabolic risk profile in overweight and obese individuals. Phys Sportsmed 2011;39:87-97.

Giada F, Vigna GB, Vitale E, Baldo-Enzi G, Bertaglia M, Crecca R, Fellin R.
Effect of age on the response of blood lipids, body composition, and aerobic power to physical conditioning and deconditioning. Metabolism 1995;44:161-165.

Hagerman FC, Walsh SJ, Staron RS, Hikida RS, Gilders RM, Murray TF, Toma K, Ragg KE. Effects of high-intensity resistance training on untrained older men. I. Strength, cardiovascular, and metabolic responses. J Gerontol A Biol Sci Med Sci 2000;55:B336-346.

Hernelahti M, Kujala UM, Kaprio J, Sarna S. Long-term vigorous training in young adulthood and later physical activity as predictors of hypertension in middle-aged and older men. Int J Sports Med 2002;23:178182.

Hinton PS, Nigh P, Thyfault J. Effectiveness of resistance training or jumping-exercise to increase bone mineral density in men with low bone mass: A 12-month randomized, clinical trial. Bone 2015;79:203212.

Hu G, Bouchard C, Bray GA, Greenway FL, Johnson WD, Newton RL Jr, Ravussin E, Ryan DH, Katzmarzyk PT. Trunk versus extremity adiposity and cardiometabolic risk factors in white and African American adults. Diabetes Care 2011;34:1415-1418.

Joseph LJ, Davey SL, Evans WJ, Campbell WW. Differential effect of resistance training on the body composition and lipoprotein-lipid profile in older men and women. Metabolism 1999;48:1474-1480.

Kettunen JA, Kujala UM, Kaprio J, Sarna S. Health of master track and field athletes: a 16-year follow-up study. Clin J Sport Med 2006;16:142148.

Kusy K, Zieliński J. Sprinters versus long-distance runners: how to grow old healthy. Exerc Sport Sci Rev 2015;43:57-64.

Kusy K, Zieliński J, Pilaczyńska-Szcześniak L. Insulin sensitivity and $\beta$-cell function estimated by HOMA2 model in sprint-trained athletes aged 20-90 years vs endurance runners and untrained participants. J Sports Sci 2013;31:1656-1664.

Law MR, Wald NJ, Thompson SG. By how much and how quickly does reduction in serum cholesterol concentration lower risk of ischaemic heart disease? BMJ 1994;308:367-372.

Leenders M, Verdijk LB, van der Hoeven L, van Kranenburg J, Nilwik R, van Loon LJ. Elderly men and women benefit equally from prolonged resistance-type exercise training. J Gerontol A Biol Sci Med Sci 2013;68: 769-779.

Martins RA, Veríssimo MT, Coelho e Silva MJ, Cumming SP, Teixeira AM. Effects of aerobic and strength-based training on metabolic health indicators in older adults. Lipids Health Dis 2010;9:76.

Mikkelsen UR, Couppé C, Karlsen A, Grosset JF, Schjerling P, Mackey AL, Klausen HH, Magnusson SP, Kjær M. Life-long endurance exercise in humans: circulating levels of inflammatory markers and leg muscle size. Mech Ageing Dev 2013;134:531-540. 
Moreira MM, Souza HP, Schwingel PA, Sá CK, Zoppi CC. Effects of aerobic and anaerobic exercise on cardiac risk variables in overweight adults. Arq Bras Cardiol 2008;91:200-206, 219-226.

Nederveen JP, Joanisse S, Séguin CM, Bell KE, Baker SK, Phillips SM, Parise G. The effect of exercise mode on the acute response of satellite cells in old men. Acta Physiol (Oxf) 2015;215:177-190.

Neves MF, Virdis A, Sanjuliani AF, Tibiriçá EV. Hypertension and cardiometabolic risk factors. Int J Hypertens 2013;2013:634798.

Nichols JF, Palmer JE, Levy SS. Low bone mineral density in highly trained male master cyclists. Osteoporos Int 2003;14:644-649.

Norton K. Sports Medicine Australia pre-exercise screening system 2005 [Internet]. Hackett: Sports Medicine Australia (SMA); 2005 [cited 2016 Aug 20]. Available from: http://sma.org.au/wp-content/uploads/2009/ 05/new_pre_screening.pdf.

Perloff D, Grim C, Flack J, Frohlich ED, Hill M, McDonald M, Morgenstern BZ. Human blood pressure determination by sphygmomanometry. Circulation 1993;88(5 Pt 1):2460-2470.

Pirillo A, Norata GD, Catapano AL. Postprandial lipemia as a cardiometabolic risk factor. Curr Med Res Opin 2014;30:1489-1503.

Reaburn P, Dascombe B. Anaerobic performance in masters athletes. Eur Rev Aging Phys 2009;6:39-53.

Reaburn P, Logan P, Mackinnon L. The effect of hypertrophy resistance training on anaerobic work capacity in veteran sprint runners. Canberra (AU): Australian Sports Commission; 1994.

Rector RS, Rogers R, Ruebel M, Hinton PS. Participation in road cycling vs running is associated with lower bone mineral density in men. Metabolism 2008;57:226-232.

Schell J, Leelarthaepin B. Physical fitness assessment in exercise and sport science. Matraville (NSW): Leelar Biomediscience Services: 1990.

Sharman JE, Stowasser M. Australian association for exercise and sports science position statement on exercise and hypertension. J Sci Med Sport 2009;12:252-257.

Sillanpää E, Laaksonen DE, Häkkinen A, Karavirta L, Jensen B, Kraemer
WJ, Nyman K, Häkkinen K. Body composition, fitness, and metabolic health during strength and endurance training and their combination in middle-aged and older women. Eur J Appl Physiol 2009;106:285296.

Sutherland WH, Woodhouse SP, Williamson S, Smith B. Decreased and continued physical activity and plasma lipoprotein lipids in previously trained men. Atherosclerosis 1981;39:307-311.

Tanner R, Gore C. Physiological tests for elite athletes. Canberra (AU): Human Kinetics; 2013.

Thompson PD, Cullinane EM, Sady SP, Flynn MM, Bernier DN, Kantor MA, Saritelli AL, Herbert PN. Modest changes in high-density lipoprotein concentration and metabolism with prolonged exercise training. Circulation 1988;78:25-34.

Treuth MS, Ryan AS, Pratley RE, Rubin MA, Miller JP, Nicklas BJ, Sorkin J, Harman SM, Goldberg AP, Hurley BF. Effects of strength training on total and regional body composition in older men. J Appl Physiol (1985) 1994;77:614-620.

Wallman K, Plant LA, Rakimov B, Maiorana AJ. The effects of two modes of exercise on aerobic fitness and fat mass in an overweight population. Res Sports Med 2009;17:156-170.

Westcott WL, Winett RA, Annesi JJ, Wojcik JR, Anderson ES, Madden PJ. Prescribing physical activity: applying the ACSM protocols for exercise type, intensity, and duration across 3 training frequencies. Phys Sportsmed 2009;37:51-58.

World Health Organization. Definition and diagnosis of diabetes mellitus and intermediate hyperglycaemia: report of a WHO/IDF consultation [Internet]. Geneva: World Health Organization; 2006 [cited 2016 Aug 20]. https://www.idf.org/webdata/docs/WHO_IDF_definition_diagnosis_of_diabetes.pdf.

Zachwieja JJ, Toffolo G, Cobelli C, Bier DM, Yarasheski KE. Resistance exercise and growth hormone administration in older men: effects on insulin sensitivity and secretion during a stable-label intravenous glucose tolerance test. Metabolism 1996;45:254-260. 